\title{
THE METHOD OF EXTREMAL LENGTH
}

\author{
BY BURTON RODIN ${ }^{1}$
}

Extremal length has become a useful tool in a wide variety of areas. The roots of the method can be traced back to the length-area comparisons in L. Ahlfors [1] and S. Warschawski [3], to the strip method of H. Grötzsch [1]-[15], and to even earlier works (see J. Jenkins [4, p. 7ff.] for a more complete historical background). In the 1940's Ahlfors and Beurling refined $^{2}$ those methods in a profound way; extremal length was introduced as a conformally invariant measure of curve families. This development appeared in Ahlfors-Beurling [4].

Subsequent applications of extremal length have been numerous and varied. There are geometric applications, function-theoretic applications, and applications which relate function-theoretic properties to geometric ones. In addition, there is a characteristically computational aspect which arises in connection with certain classical problems on univalent functions. I wish to discuss examples in each of these areas. There are many important topics I shall omit (prime ends, quasiconformal mapping, generalized modulus, and generalized capacity to mention a few); the extended bibliography will give a more complete picture.

1. The concept of extremal length. Let $R$ be a surface (a surface, here, will be required to be connected, orientable, and to satisfy the second axiom of countability) with Riemannian metric $d s_{0}$. An important special case will be a region $R$ in the plane endowed with the Euclidean metric.

A second metric $d s$ on $R$ is said to be conformally equivalent to $d s_{0}$ if these two metrics give rise to the same angular measure in each tangent space of $R$. This condition is also expressed by the requirement that at each point of $R$ the metrics are proportional: $d s=\rho d s_{0}$ where $\rho$ is a positive function on $R$.

An invited address presented to the 705th Meeting of the Society in Bellingham, Washington on June 16, 1973; received by the editors October 1, 1973.

AMS (MOS) subject classifications (1970). Primary 30A40; Secondary 30A52, 30A30.

Key words and phrases. Extremal length, conformal invariants, conformal mapping, Riemann surfaces.

${ }^{1}$ Research partially supported by the National Science Foundation grant No. GP 38600.

${ }^{2}$ For a glimpse of this development through the eyes of one of the principals, see the recent book L. Ahlfors [9, pp. 50, 81].

Copyright @ American Mathematical Society 1974 
We shall define extremal length in a manner which emphasizes the conformal invariance. Let $\Gamma$ be a family of curves on $R$. For each metric $d s$ on $R$, define the ds-length of $\Gamma$ to be

$$
L(\Gamma, d s)=\inf _{\gamma \in \Gamma} \int_{\gamma} d s .
$$

The area of $R$ in the metric $d s$ will be denoted by $A(d s)$. Except for the regularity conditions that will be made explicit in a moment, the extremal length of the family $\Gamma$ will be defined by

$$
\lambda(\Gamma)=\sup \left(L^{2}(\Gamma, d s) / A(d s)\right),
$$

where the supremum is taken over all metrics $d s$ which are conformally equivalent to $d s_{0}$.

It is clear from the definition that $\lambda(\Gamma)$ depends only on the angular measurements induced by the original metric $d s_{0}$, and not on the metric itself. Thus the definition can be applied if $R$ is any Riemann surface. In addition, we see immediately that the following theorem on conformal invariance is valid.

THEOREM 1 (CONFORMAL INVARIANCE OF EXTREMAL LENGTH). Let $f$ be $a$ 1-1 conformal mapping of a Riemann surface $R$ onto a Riemann surface $S$. Let $\Gamma$ be a family of curves on $R$. Then $\lambda(\Gamma)=\lambda(f(\Gamma))$.

For constructions and estimations, extremal length will be most flexible when the regularity conditions on $d s$ are weakest. On the other hand, the integrals in (1) and $A(d s)$ impose some degree of regularity. A number of different conditions have been suggested in the literature; see M. Ohtsuka $[9$, p. $70 \mathrm{ff}$.$] , for a survey. A very satisfactory requirement, and the one$ I shall adopt, is the following: the supremum in (2) is taken over all $d s$ of the form $d s=\rho d s_{0}$, where $\rho$ is a nonnegative Borel measurable function on $R$. This insures the existence of both integrals $\int_{\gamma} d s$ and $A(d s)$, although they might be infinite. In the definition (2) we also set $0 / 0=\infty / \infty=0$. It should be noted that $d s$ is not necessarily a Riemannian metric in the usual sense-it may have zeros, and it need not be differentiable. Such a $d s$ is called a linear density. The conformal invariance theorem, Theorem 1, still holds. In fact, we have (M. Ohtsuka [9, pp. 80-84]):

THEOREM 2. Let $f: R \rightarrow S$ be a complex analytic mapping of Riemann surfaces. Let $\Gamma$ be a curve family on $R$. Then $\lambda(\Gamma) \leqq \lambda(f(\Gamma))$.

\{An alternate definition of extremal length given in L. Ahlfors [6] admits competing metrics $d s$ which need not be conformally equivalent to $d s_{0}$. If $d s$ is any Riemannian metric on $R$, the ratio $d s / d s_{0}$ has a maximum $\lambda_{1}$ and a minimum $\lambda_{2}$ at each point of $R$. The dilatation $K$ is defined as 
$K=\lambda_{1} / \lambda_{2}$. It is proved that $\lambda(\Gamma)=\sup \left(L^{2}(\Gamma, d s) / A^{\prime}(d s)\right)$, where $A^{\prime}(d s)=$ $\iint_{R} K d \omega$ and $d \omega$ is the area element in the metric $\left.d s.\right\}$

In certain simple cases extremal length can be computed explicitly. Consider a rectangle $R$ in the plane, $R=\{(x, y) \mid 0<x<a, 0<y<b\}$. Let $\Gamma$ consist of all arcs in $R$ which join the sides of length $b$. A lower bound for $\lambda(\Gamma)$ is obtained by computing $L^{2}(\Gamma, d s) / A(d s)$ for any specific $d s$; if we use the Euclidean metric $|d z|$ we obtain the lower bound

$$
\lambda(\Gamma) \geqq L^{2}(\Gamma,|d z|) / A(|d z|)=a^{2} / a b=a / b .
$$

An upper bound can be found by choosing a subfamily of $\Gamma$. The horizontal segments, $\gamma_{y}: t \mapsto(t, y)$ for $0<t<a$, yield

$$
L^{2}(\Gamma, \rho|d z|) \leqq\left[\int_{\gamma_{\nu}} \rho|d z|\right]^{2} \leqq\left[\int_{\gamma_{y}} \rho^{2}|d z|\right]\left[\int_{\gamma_{\nu}}|d z|\right]=a \int_{\gamma_{\nu}} \rho^{2} d x
$$

integrate for $y \in(0, b)$ and obtain

$$
b L^{2}(\Gamma, \rho|d z|) \leqq a \iint_{R} \rho^{2} d x d y=a A(\rho|d z|) .
$$

Thus $L^{2}(\Gamma, \rho|d z|) / A(\rho|d z|) \leqq a \mid b$, and since $\rho$ was arbitrary $\lambda(\Gamma) \leqq a \mid b$. This proves

THEOREM 3. Let $R$ be a rectangle of sides $a$ and $b$. Let $\Gamma$ be the family of arcs in $R$ which join the sides of length $b$. Then $\lambda(\Gamma)=a \mid b$.

Two other explicit values for extremal lengths $d s$ are given in the following elementary theorem.

THEOREM 4. Let $R$ be the annulus $a<|z|<b$. Let $\Gamma$ be the family of arcs in $R$ which join the two contours. Let $\Gamma^{*}$ be the family of closed curves in $R$ which separate the two contours. Then $\lambda(\Gamma)=\lambda^{-1}\left(\Gamma^{*}\right)=(1 / 2 \pi) \log (b / a)$.

2. Geometric inequalities. There are a number of purely geometric applications of extremal length. The simplest example concerns the general quadrilateral (a Jordan curve with four distinguished points).

THEOREM 5. Let $R$ be a general quadrilateral of area $A$. Let a be the length of the shortest arc in $R$ connecting one pair of opposite sides. Let $b$ be the length of the shortest arc in $R$ connecting the other pair of sides. Then $a \cdot b \leqq A$.

A purely geometric proof of this theorem (E. Rengel [1], O. Teichmüller [1]) was given by A. Besicovitch [1] in response to a functiontheoretic proof presented to him by Charles Löwner. The geometric proof is difficult. The use of extremal length, however, makes the proof trivial. 
Let $\Gamma$ be the family of arcs in $R$ which join one pair of opposite sides, and let $\Gamma^{*}$ be the family of arcs in $R$ which join the other two sides. There is a conformal map $f$ of $R$ onto some rectangle such that $f(\Gamma)$ and $f\left(\Gamma^{*}\right)$ are the families of arcs which join opposite sides of the rectangle. By Theorem 3, $\lambda(f(\Gamma)) \cdot \lambda\left(f\left(\Gamma^{*}\right)\right)=1$. By the conformal invariance, $\lambda(\Gamma) \cdot \lambda\left(\Gamma^{*}\right)=1$. The Euclidean metric $|d z|$ in $R$ can be used to give lower bounds for these latter two extremal lengths. Thus

$$
\frac{a^{2}}{A} \cdot \frac{b^{2}}{A}=\frac{L^{2}(\Gamma,|d z|)}{A(|d z|)} \cdot \frac{L^{2}\left(\Gamma^{*},|d z|\right)}{A(|d z|)} \leqq \lambda(\Gamma) \cdot \lambda\left(\Gamma^{*}\right)=1,
$$

and the theorem follows at once.

There is a generalization of Theorem 5 to higher connectivity (Rodin [7]). Let $E$ be a compact set contained in the interior of the quadrilateral $R$, and suppose that $R_{1}=R-E$ is connected. Theorem 5 would be false if we replaced $R$ by $R_{1}$. It is possible to generalize from $R$ to $R_{1}$ if the definitions of $a$ and $b$ are suitably generalized. Let $\Gamma_{1}$ be the family of arcs in $R_{1}$ which join one pair of opposite sides. Let $\Gamma_{1}^{*}$ be the family of curves (a curve is to mean a union of arcs) in $R_{1}$ which separate that pair of sides. An extremal length theorem asserts that $\lambda\left(\Gamma_{1}\right) \cdot \lambda\left(\Gamma_{1}^{*}\right)=1$. It follows immediately that if $a$ is the infimum of the lengths of $\Gamma_{1}$, and if $b$ is the infimum of the lengths of $\Gamma_{1}^{*}$, then $a \cdot b \leqq A$, where $A$ is the area of $R_{1}$.

The extremal lengths $\lambda\left(\Gamma_{1}\right), \lambda\left(\Gamma_{1}^{*}\right)$ considered above play an important role in characterizing certain function-theoretic properties of the set $E$. We shall return to such applications in $\$ 5$.

Here is another geometric application of extremal length (O. Teichmüller, C. Löwner, P. M. Pu [1], L. Keen [1], B. Rodin [7]):

THEOREM 6. Let $R$ be a torus with a Riemannian metric. Let a be the length of the shortest nontrivial cycle on $R$, and let $A$ be the area of $R$. Then $a^{2} \leqq 2 A / \sqrt{ } 3$.

This theorem can be proved easily by first mapping $R$ conformally onto a flat torus represented by a parallelogram $0,1, \tau, 1+\tau$ in the plane. One can always choose $\tau$ to be in a fundamental region of the modular group, so we may assume $-\frac{1}{2} \leqq \operatorname{Re} \tau \leqq \frac{1}{2},|\tau| \geqq 1, \operatorname{Im} \tau>0$. Thus $\operatorname{Im} \tau \geqq \sqrt{ } 3 / 2$. In the parallelogram, the extremal length of the horizontal line segments is easily seen to be $1 / \operatorname{Im} \tau$. Therefore the corresponding cycles $\Gamma$ on the torus have extremal length $1 / \operatorname{Im} \tau$. Therefore, if $d s_{0}$ is the original metric on the torus then $L^{2}\left(\Gamma, d s_{0}\right) / A\left(d s_{0}\right) \leqq 2 / \sqrt{ } 3$ for these particular cycles $\Gamma$, and the theorem follows.

The following theorem was proved by C. Blatter [1]. It is a profound generalization of Theorem 6, although the constant is no longer best possible. 
THEOREM 7. Let $R$ be a compact surface of genus $g$ with a Riemannian metric. Let $a$ be the length of the shortest nontrivial cycle on $R$. Let $A$ be the area of $R$. Then

$$
a^{2}<(2 / \pi)((g+1) !)^{1 / g} A .
$$

The proof of Theorem 7 is too long to repeat here. However, it will be useful to sketch the main ideas in order to point out an important extremal length result which arose in the course of the proof.

There is a linear mapping (de Rham, Hodge) of the 1-cycles on $R$ to the harmonic 1-forms on $R$. The harmonic differential $\sigma_{c}$ which corresponds to the 1-cycle $c$ under this mapping satisfies the two properties:

$$
\begin{gathered}
\int_{c} \omega=\left(\omega, \sigma_{c}\right) \text { for all closed 1-forms } \omega, \\
\int_{d}^{*} \sigma_{c}=c \times d \text { for all 1-cycles } d,
\end{gathered}
$$

where $\left(\omega, \sigma_{c}\right)=\iint_{R} \omega \wedge * \sigma_{c}$ is the inner product, and $c \times d$ is the intersection number.

The norm $\left(\sigma_{c}, \sigma_{c}\right)^{1 / 2}$ can be related to extremal length. Let $\lambda([c])$ denote the extremal length of the homology class of $c$. C. Blatter [1] and R. Accola [1] proved independently that

$$
\lambda([c])=\left(\sigma_{c}, \sigma_{c}\right) .
$$

The important result (5) (it will be discussed in more detail later) shows that once a homology basis $c_{1}, c_{2}, \cdots, c_{2 g}$ is chosen for the 1-cycles, the $2 g \times 2 g$ matrix $S=\left\|\left(\sigma_{c_{2}}, \sigma_{c_{j}}\right)\right\|$ determines the extremal lengths $\lambda([c])$ of all homology classes [c] on $R$ by means of

$$
\lambda\left(\left[\sum n_{i} c_{i}\right]\right)=\sum n_{i} n_{j}\left(\sigma_{c_{i}}, \sigma_{c_{j}}\right)=n S n^{t} .
$$

There is a theorem of Minkowski which guarantees, for any positive definite $2 g \times 2 g$ matrix $Q$ of determinant 1 , the existence of a nonzero integer vector $\boldsymbol{m}$ which satisfies $\boldsymbol{m} Q \boldsymbol{m}^{t} \leqq(2 / \pi)[(g+1) !]^{1 / g}$. The next part of Blatter's proof consists in showing that the matrix $S$ satisfies the hypotheses of Minkowski's theorem if the homology basis $c_{1}, c_{2}, \cdots, c_{2 g}$ is suitably chosen. After that is accomplished the vector $\boldsymbol{m}=\left(m_{1}, m, \cdots, m_{2 g}\right)$ can be used to form a nontrivial $c=m_{1} c_{1}+m_{2} c_{2}+\cdots+m_{2 g} c_{2 g}$ with $\lambda([c]) \leqq$ $(2 / \pi)[(g+1) !]^{1 / g}$. Therefore, if $d s_{0}$ is the original metric on $R$ then $L^{2}\left([c], d s_{0}\right) / A\left(d s_{0}\right) \leqq(2 / \pi)[(g+1) !]^{1 / g}$ and the theorem follows.

The Accola-Blatter result (5) has interesting ramifications. For example, it shows that the matrix $S$ is completely determined by the extremal lengths of a finite number of homology classes. The matrix $S$, in turn, determines 
the Riemann period matrix of the compact Riemann surface underlying $R$. Hence, by Torelli's theorem, we conclude that a finite number of extremal lengths of homology classes provide a complete set of conformal invariants for compact Riemann surfaces. The same is true for plane regions of finite connectivity.

Other ramifications of (5) involve noncompact Riemann surfaces. Accola [1] proved (5) for an arbitrary $R$, compact or not. A. Marden [1] and Rodin [1] considered other kinds of homology classes. Let $[c]_{1}$ denote the homology classes of $c$ modulo dividing cycles. Let $[c]_{2}$ denote the relative homology class of $c$ (chains may have infinitely many simplexes). Marden [1] proved that $\lambda\left([c]_{2}\right)=\left(\sigma_{h 0}, \sigma_{h 0}\right)$; Rodin [1] proved that $\lambda\left([c]_{1}\right)=$ $\left(\sigma_{h s e}, \sigma_{h s e}\right)$. Here $\sigma_{h 0}$ and $\sigma_{h s e}$ are the projections of $\sigma_{c}$ onto the subspaces $\Gamma_{h 0}$ and $\Gamma_{h s e}$ of the Hilbert space $\Gamma_{h}$ of square integrable 1-forms on $R$. The subspace $\Gamma_{h 0}$ is the orthogonal complement of $\Gamma_{h e}^{*}$, where $\Gamma_{h e}$ is the space of exact harmonic 1-forms. The subspace $\Gamma_{h s e}$ consists of the semiexact harmonic 1-forms.

Another generalization of (5) was recently obtained by C. D. Minda [2]. He was able to relate the norm $\left(\sigma_{c}, \sigma_{c}\right)$ to extremal length in the case that $c$ is an arbitrary 1-chain, not necessarily a cycle. This result is especially interesting if $R$ is a compact Riemann surface, for it leads to an extremal length characterization of principal divisors-a geometric counterpart to the classical theorem of Abel.

Extremal length has been used to obtain length-area inequalities related to flows. Let $c$ be a 1-cycle on a compact Riemannian 2-space $R$. Then, in the sense of F. Klein [1], there is a steady fluid flow on $R$ corresponding to $c$; namely, the flow which has the property that along any closed curve $d$ the change in the potential is the intersection number $c \times d$. Let a be the length of the shortest geodesic homologous to $c$. Let $b$ be the length of the shortest geodesic, not necessarily a cycle, along which the potential drop is 1 . Then $a \cdot b \leqq A$, where $A$ is the area of $R$ (Rodin [7]).

3. Infinite extremal length. Having discussed some geometric applications of extremal length, we turn next to applications in the area of analysis. Just as measure theory provides the notion of a negligible point set (measure zero), extremal length provides the notion of a negligible curve family. Following B. Fuglede [1], we say that a property holds for almost all curves in a curve family $\Gamma$ if the subfamily of exceptional curves has infinite extremal length. (In this context the reciprocal of the extremal length, called the modulus, may be more natural.) This notion may be justified somewhat by the following properties of infinite extremal length: For any curve families $\Gamma, \Gamma_{0}, \Gamma_{1}, \cdots$

$$
\lambda\left(\Gamma_{0}\right)=\infty \Rightarrow \lambda\left(\Gamma \cup \Gamma_{0}\right)=\lambda(\Gamma)
$$


and

$$
\lambda\left(\Gamma_{n}\right)=\infty \text { for } n=1,2, \cdots \Rightarrow \lambda\left(\cup \Gamma_{n}\right)=\infty .
$$

Some examples may help to illustrate the property of infinite extremal length. Consider the horizontal line segments $\gamma_{y}=\{(x, y) \mid 0 \leqq x \leqq 1\}$, and let $\Gamma=\left\{\gamma_{y} \mid y \in E\right\}$ where $E$ is a measurable set of real numbers. Then $\lambda(\Gamma)=\infty$ if and only if $E$ has measure zero.

As a second example, let $E$ be a compact point set in $|z|<1$, and let $\bar{\Gamma}$ consist of all arcs which join $|z|=1$ to $E$. Then $\lambda(\Gamma)=\infty$ if and only if the logarithmic capacity of $E$ vanishes.

There are many function-theoretic applications of this notion of infinite extremal length. A striking example of its usefulness occurs in the problem of the existence of boundary values for functions with a finite Dirichlet integral. For simplicity, consider the case of a $C^{1}$-function $u$ defined in a plane region $R$. The Dirichlet integral of $u$ is $D(u)=\iint_{R}\left(u_{x}^{2}+u_{y}^{2}\right) d x d y$. We say that an arc $\gamma:[0,1) \rightarrow R$ in $R$ tends to the boundary of $R$ if $\bigcap\{\mathrm{Cl} \gamma[(t, 1)] \mid 0 \leqq t<1\} \subset \partial R$. The next theorem (M. Ohtsuka [8], [9]) shows that, in a general sense, $u$ has boundary values if $D(u)<\infty$. The significant point is that no assumptions whatever are required concerning the geometric boundary of $R$.

THEOREM 8. If $D(u)<\infty$ then $u$ has a limit along almost all arcs in $R$ which tend to the boundary.

An extremal length argument makes the proof trivial. Consider the linear density $d s=|\operatorname{grad} u||d z|$. Then $A(d s)=D(u)<\infty$. Let $\Gamma$ be the family of arcs in $R$ which tend to the boundary. The subfamily $\Gamma^{\prime}=$ $\left\{\gamma \in \Gamma \mid \int_{\gamma} d s=\infty\right\}$ clearly has infinite extremal length. If $\gamma \in \Gamma-\Gamma^{\prime}$ then $\int_{\gamma} d s<\infty$, and hence $\int_{\gamma}|d u|<\infty$. Thus $u$ has a limit along each such $\gamma$, and the theorem is proved.

In the situation of Theorem 8 , suppose $u$ has a limit along two arcs which tend to the same boundary point of $R$. The two limiting values would not, in general, be expected to agree; for example, the arcs might tend to different prime ends. The next result (Ohtsuka [9]) gives a useful condition for equality of these limits.

THEOREM 9. Let $c_{1}$ and $c_{2}$ be arcs with a common terminal point $z_{0}$, and suppose they form the boundary of a Jordan region $\Omega$. Let $u$ be continuous in $\mathrm{Cl} \Omega-\left\{z_{0}\right\}$ and satisfy $D(u)<\infty$. If $u$ tends to a limit a along $c_{1}$, and to a limit $b$ along $c_{2}$, then $a=b$.

Again, extremal length provides a simple method of proof. Consider the family of circular cross cuts of $\Omega$ with center at $z_{0}$ and radius $\leqq \varepsilon$. It is elementary that $\lambda(\Gamma)=0$. Therefore the density $d s=|\operatorname{grad} u||d z|$ satisfies $\inf _{\gamma \in \Gamma} \int_{\gamma} d s=0$, and hence $\inf _{\gamma \in \Gamma} \int|d u|=0$. It follows that $a=b$. 
An interesting consequence of Theorem 9 is that the boundary values of a Dirichlet finite function cannot have a jump discontinuity if the region is bounded by Jordan curves. Other types of discontinuities are possible. Further results on the boundary values of Dirichlet finite functions, though not using the method of extremal length, are contained in J. Doob [1] and J. L. Lions and E. Magenes [1].

M. Ohtsuka [8], [9] uses extremal length to develop a very general form of Dirichlet's principle. His result, Theorem 10 below, shows that this notion of almost all curves describes perfectly the mode in which a harmonic function can be made to imitate the boundary behavior of a Dirichlet finite function.

TheOREM 10. Let u be a $C^{1}$-function on a hyperbolic Riemann surface $R$ with $D(u)<\infty$. Then there is a unique harmonic function $U$ such that $U-u$ has limit zero along almost all arcs in $R$ which tend to the boundary.

The notion of almost all curves has interesting applications to extremal length itself. This will be discussed next.

4. Extremal metrics. Suppose $\Gamma$ is a curve family on a Riemann surface $R$, and $0<\lambda(\Gamma)<\infty$. We are interested in the problem of existence and uniqueness for an extremal metric, that is, a metric $d s$ which satisfies $\lambda(\Gamma)=L^{2}(\Gamma, d s) / A(d s)$. Simple examples show that an extremal metric need not exist in general. In the following discussion Theorems 11, 12, and 13 are based on K. Strebel [2] and N. Suita [3].

Consider the set $P(\Gamma)$ consisting of all linear densities $d s$ on $R$ which satisfy $A(d s)<\infty$ and $L(\Gamma, d s) \geqq 1$. Thus $\lambda^{-1}(\Gamma)=\inf \{A(d s) \mid d s \in P(\Gamma)\}$. Note that $P(\Gamma)$ is a convex subset of a Hilbert space ${ }^{3}$ where $\sqrt{ } A(d s)$ is the norm. Therefore $\lambda^{-1}(\Gamma)=A\left(d s_{0}\right)$ for the unique linear density $d s_{0}$ of minimum norm in the closure $\mathrm{Cl} P(\Gamma)$ of $P(\Gamma)$. The following theorem characterizes the linear densities in $\mathrm{Cl} P(\Gamma)$.

THEOREM 11. $\mathrm{Cl} P(\Gamma)$ consists of all nonnegative linear densities $d s$ such that $\int_{\gamma} d s \geqq 1$ for almost all $\gamma \in \Gamma$.

We can now answer the question about extremal metrics.

THEOREM 12. Let $0<\lambda(\Gamma)<\infty$. There is a linear density $d s_{0}$ which is the extremal metric for a subfamily $\Gamma_{0} \subset \Gamma$. $\Gamma_{0}$ satisfies $\lambda\left(\Gamma-\Gamma_{0}\right)=\infty$, and hence $\lambda\left(\Gamma_{0}\right)=\lambda(\Gamma)$. The family $\Gamma$ determines $d s_{0}$ uniquely (a.e.) up to a positive multiplicative constant.

\footnotetext{
${ }^{3}$ The Hilbert space consists of square integrable densities $\rho|d z|$ where the functions $\rho$ are no longer required to be nonnegative.
} 
Let us call the metric $d s_{0}$ of Theorem 12 the generalized extremal metric for $\Gamma$. It satisfies the following inequality, a consequence of the "law of cosines":

THEOREM 13. If $d s \in \mathrm{Cl} P(\Gamma)$, and if $d s_{0}$ is the generalized extremal metric for $\Gamma$, then $A\left(d s_{0}\right) \leqq A(d s)-A\left(d s-d s_{0}\right)$.

Next, I wish to present an explicit method for finding the extremal metric. The method is restricted to one-parameter curve families. However, it has much wider applicability because it can often be applied to oneparameter subfamilies of a general curve family (see $\$ 6$ below).

For each $s \in(a, b)$, suppose $c_{s}: t \mapsto c_{s}(t)$ is a curve in $R$. The domain of $c_{s}$ may depend on $s$; suppose it is an open interval $t_{\mathrm{c}}(s)<t<t_{1}(s)$. We shall assume that $R$ is a region in the $x, y$-plane, and that the mapping $(s, t) \rightarrow$ $c_{s}(t)=(x, y)$ is $1-1$ and has positive Jacobian determinant $\partial(x, y) / \partial(s, t)$. Then the extremal length of the curve family $\Gamma=\left\{c_{s}\right\}_{a<s<b}$ can be calculated exactly:

THEOREM 14. Let

$$
l(s)=\int_{t_{0}(s)}^{t_{1}(s)} \frac{\left|d c_{s}(t) / d t\right|^{2}}{\partial(x, y) / \partial(s, t)} d t .
$$

Then

$$
\frac{1}{\lambda(\Gamma)}=\int_{a}^{b} \frac{d s}{l(s)} .
$$

Theorem 14 can be considered as a generalization of the known formulas for calculating the extremal length of a family of parallel line segments, concentric circular arcs, and line segments on a pencil (cf. Theorems 2.4 and 2.6 in M. Ohtsuka [9]). Integrals of the form (10) appeared in Ahlfors [1] and Warschawski [1]. I have not determined the weakest regularity conditions which would justify the following computations.

For the proof of Theorem 14, define

$$
\rho_{0}\left(c_{s}(t)\right)=\frac{1}{l(s)} \cdot \frac{\left|d c_{s}(t) / d t\right|}{\partial(x, y) / \partial(s, t)}
$$

where $l(s)$ is given by (9). We shall show that $\rho_{0}(z)|d z|$ is the extremal metric for $\Gamma$. We first note that $\int_{c_{s}} \rho_{0}|d z|=1$ for each $c_{s}$ :

$$
\begin{aligned}
\int_{c_{s}} \rho_{0}(z)|d z| & =\int_{t_{0}(s)}^{t_{1}(s)} \rho_{0}\left(c_{s}(t)\right)\left|\frac{d}{d t} c_{s}(t)\right| d t \\
& =\int_{t_{0}(s)}^{t_{1}(s)} \frac{1}{l(s)} \cdot \frac{\left|d c_{s}(t) / d t\right|^{2}}{\partial(x, y) / \partial(s, t)} d t=\frac{1}{l(s)} \cdot l(s)=1 .
\end{aligned}
$$


Next, suppose that $\rho(z)|d z|$ is any linear density which satisfies $\int_{c_{s}} \rho(z)|d z| \geqq 1$ for each $c_{s}$. Then for each $s$

and so

$$
\begin{array}{r}
\int_{c_{s}}\left[\rho(z)-\rho_{0}(z)\right]|d z| \geqq 0, \\
\int_{t_{0}(s)}^{t_{1}(s)}\left[\rho\left(c_{s}(t)\right)-\rho_{0}\left(c_{s}(t)\right)\right]\left|\frac{d}{d t} c_{s}(t)\right| d t \geqq 0,
\end{array}
$$

$$
\int_{t_{0}(s)}^{t_{1}(s)} \frac{1}{l(s)}\left[\rho\left(c_{s}(t)\right)-\rho_{0}\left(c_{s}(t)\right)\right]\left|\frac{d}{d t} c_{s}(t)\right| d t \geqq 0 .
$$

We integrate (12) for $s \in(a, b)$ and write the resulting inequality in the form

$$
\iint_{S}\left[\rho\left(c_{s}(t)\right)-\rho_{0}\left(c_{s}(t)\right)\right] \rho_{0}\left(c_{s}(t)\right) \frac{\partial(x, y)}{\partial(s, t)} d s d t \geqq 0,
$$

which gives $\iint_{R} \rho(z) \rho_{0}(z) d x d y \geqq \iint_{R} \rho_{0}^{2}(z) d x d y$. By use of the Schwarz inequality we then arrive at

$$
\iint_{R} \rho^{2} d x d y \geqq \iint_{R} \rho_{0}^{2} d x d y .
$$

Equation (13) shows that $\rho_{0}|d z|$ is the extremal metric. It can be used to calculate the extremal length:

$$
\begin{aligned}
\frac{1}{\lambda(\Gamma)} & =\iint_{R} \rho_{0}^{2} d x d y=\iint_{S} \rho_{0}^{2}\left(c_{s}(t)\right) \frac{\partial(x, y)}{\partial(s, t)} d s d t \\
& =\int_{a}^{b} \int_{t^{0}(s)}^{t_{1}(s)} \frac{\left|d c_{s}(t) / d t\right|^{2}}{\partial(x, y) / \partial(s, t)} d t \frac{d s}{l^{2}(s)}=\int_{a}^{b} l(s) \frac{d s}{l^{2}(s)}=\int_{a}^{b} \frac{d s}{l(s)},
\end{aligned}
$$

and we arrive at (10). As a corollary of the proof we obtain

THEOREM 15. In the situation of Theorem 14, the extremal metric for $\Gamma$ is

where $z=c_{s}(t)$.

$$
\rho_{0}(z)|d z|=\frac{1}{l(s)} \frac{\left|d c_{s}(t) / d t\right|}{\partial(x, y) / \partial(s, t)}|d z|
$$

5. Extremal distance and function theoretic null-sets. Extremal length provides a link for relating geometric and function-theoretic quantities. This aspect can be illustrated by the several notions of extremal distance. 
Let $s_{1}$ and $s_{2}$ be disjoint subsets contained either in a Riemann surface $R$ or on its border. Let $\Gamma$ be the family of arcs in $R$ which join $s_{1}$ and $s_{2}$. The extremal length of $\Gamma$ is then called the extremal distance between $s_{1}$ and $s_{2}$, and it is denoted by $\lambda\left(s_{1}, s_{2}\right)$.

We can modify this definition by considering other ways in which a curve might be said to "join" $s_{1}$ and $s_{2}$. For example, consider the Kerékjártó-Stoïlow compactification $R_{K}$ of $R ; R_{K}$ is obtained by adding to $R$ an ideal point for each boundary component of $R$. An arc on $R_{K}$ determines a curve on $R$. If an arc on $R_{K}$ joins $s_{1}$ to $s_{2}$, the corresponding curve on $R$ might be said to join $s_{1}$ to $s_{2}$ "via the boundary components". Let $\Gamma_{K}$ consist of all arcs on $R_{K}$ which join $s_{1}$ to $s_{2}$, and let $\lambda_{K}\left(s_{1}, s_{2}\right)$ be the extremal length of the curve family on $R$ determined by $\Gamma_{K}$. Then $\lambda_{K}\left(s_{1}, s_{2}\right)$ is another notion of extremal distance between $s_{1}$ and $s_{2}$.

A third notion of extremal distance is obtained by considering the onepoint compactification $R_{\infty}$ of $R$. An arc on $R_{\infty}$ which joins $s_{1}$ to $s_{2}$ determines a curve on $R$ which joins $s_{1}$ to $s_{2}$ "via the ideal boundary point". Let $\Gamma_{\infty}$ consist of all arcs on $R_{\infty}$ which join $s_{1}$ to $s_{2}$, and let $\lambda_{\infty}\left(s_{1}, s_{2}\right)$ denote the extremal length of the corresponding curve family on $R$.

Note that $\Gamma \subset \Gamma_{K} \subset \Gamma_{\infty}$ when we consider these as curve families on $R$. Therefore the three notions of extremal distance satisfy

$$
\lambda\left(s_{1}, s_{2}\right) \geqq \lambda_{K}\left(s_{1}, s_{2}\right) \geqq \lambda_{\infty}\left(s_{1}, s_{2}\right) .
$$

The conditions for equality in (14) are related to function theoretic nullsets:

THEOREM 16. Let $R$ be a plane region with complement $E$. The logarithmic capacity of $E$ vanishes if and only if $\lambda\left(s_{1}, s_{2}\right)=\lambda_{\infty}\left(s_{1}, s_{2}\right)$ for every pair of disjoint closed disks $s_{1}, s_{2}$ contained in $R$.

THEOREM 17. Let $R$ be a plane region with complement $E$. The set $E$ is a removable singularity for every Dirichlet finite analytic function if and only if $\lambda\left(s_{1}, s_{2}\right)=\lambda_{K}\left(s_{1}, s_{2}\right)$ for every pair of disjoint closed disks $s_{1}, s_{2}$ contained in $R$.

Theorems 16 and 17 can be generalized to Riemann surfaces merely by replacing the conditions relating to $E$ by the intrinsic conditions $R \in O_{H D}$ in Theorem 16, and $R \in O_{K D}$ in Theorem 17. Theorem 17 was proved in Rodin [4], and Theorem 16 was proved in Minda [1]. The earliest result of this type is due to Ahlfors-Beurling [4]; an extension to higher dimensions is given in $J$. Väisälä [3].

6. The classical origins. It has already been mentioned that extremal length had its roots in the techniques used over forty years ago to investigate certain problems in complex analysis. It is of interest to see if the modern theory of extremal length can be profitably reapplied to those 
original problems. A program of this sort was recently begun by J. Jenkins-K. Oikawa [6] (cf. also D. Gaier [1] and A. Obrock [1]). In addition to improving a result of Hayman, Jenkins and Oikawa showed that extremal length methods provide significant simplifications in the proofs of the fundamental inequalities of Ahlfors.

One of the original problems was the behavior of the Riemann mapping function near a boundary point of the domain. Ahlfors introduced the useful normalization of applying a logarithmic transformation to the domain and range. The result is a conformal mapping $w=f(z)$ of a strip domain $S$ onto a parallel strip. As $z$ tends to a certain infinite boundary element, $f(z)$ tends to $+\infty$.

Suppose that the strip domain $S$ is determined by the graphs of two real valued functions $y=\varphi_{-}(x)$ and $y=\varphi_{+}(x)$ :

$$
S=\left\{z=x+i y \mid \varphi_{-}(x)<y<\varphi_{+}(x)\right\} .
$$

The conformal mapping $w=f(z)$ sends $S$ onto a parallel strip $R=$ $\{w=u+i v \mid-\pi / 2<v<\pi / 2\}$, and $f(z) \rightarrow+\infty$ as $z \rightarrow+\infty, z \in S$. There are a number of problems of interest in this situation. For example, what properties of $\varphi_{-}$and $\varphi_{+}$guarantee the existence of $\lim _{z \rightarrow+\infty}[f(z)-z]$ (the problem of differentiability at the boundary)?

In their extremal length formulation, these kinds of questions lead to the problem of estimating extremal distance in a quadrilateral. Let $Q=$ $Q\left(x_{0}, x_{1}\right)$ be the quadrilateral region $\left\{z=x+i y \mid z \in S, x_{0}<x<x_{1}\right\}$. Let $M=M\left(x_{0}, x_{1}\right)$ be the extremal length of all arcs in $Q$ which join the vertical sides of $Q$. The crucial problem is to obtain useful estimates of $M$.

Theorem 14 is useful for this purpose. It gives the exact extremal length of a one-parameter curve family. If $\Gamma$ is any one-parameter family of arcs in $Q$ which join the vertical sides, then $\lambda(\Gamma)$ serves as an upper bound for $M$. If $\Gamma^{*}$ is a one-parameter family of arcs in $Q$ which separate the vertical sides, then $\lambda^{-1}\left(\Gamma^{*}\right)$ serves as a lower bound for $M$. With judicious choices of $\Gamma$ and $\Gamma^{*}$, useful bounds on $M$ can be obtained. (The bounds will be exact if $\Gamma$ and $\Gamma^{*}$ correspond to the coordinate grid in the rectangular conformal image of $Q$.)

An obvious first choice for $\Gamma$ is the family $\left\{c_{s}\right\}_{0<s<1}$, where $c_{s}(t)=$ $t+i\left[s \varphi_{-}(t)+(1-s) \varphi_{+}(t)\right]$ for $x_{1}<t<x_{2}$. An application of Theorem 14 yields

$$
\begin{aligned}
l(s) & =\int_{x_{1}}^{x_{2}} \frac{1+\left[s \varphi_{-}^{\prime}(t)+(1-s) \varphi_{+}^{\prime}(t)\right]^{2}}{\varphi_{+}(t)-\varphi_{-}(t)} d t, \\
\lambda(\Gamma) & =\left\{\int_{0}^{1} \frac{d s}{l(s)}\right\}^{-1} \leqq \int_{0}^{1} l(s) d s \\
& =\int_{x_{1}}^{x_{2}} \frac{3+\varphi_{+}^{\prime 2}(t)+\varphi_{+}^{\prime}(t) \varphi_{-}^{\prime}(t)+\varphi_{-}^{\prime 2}(t)}{3\left[\varphi_{+}(t)-\varphi_{-}(t)\right]} d t .
\end{aligned}
$$


Thus the integral in (15) is an upper bound for $M$. It is essentially the same as the one derived by Jenkins-Oikawa [6]; we have derived it in a different way.

An obvious first choice for $\Gamma^{*}$ is the family $\left\{c_{s}\right\}$ of vertical line segments $c_{s}(t)=s+i t\left(\varphi_{-}(s)<t<\varphi_{+}(s)\right)$. The calculation of $\lambda\left(\Gamma^{*}\right)$ yields

$$
\frac{1}{\lambda\left(\Gamma^{*}\right)}=\int_{x_{1}}^{x_{2}} \frac{d t}{\varphi_{+}(t)-\varphi_{-}(t)} .
$$

The integral in (16) is therefore a lower bound for $M$. It also is the same as the lower bound derived by Jenkins-Oikawa.

Substantial improvements in the bounds (15) and (16) can be obtained from more sophisticated choices of $\Gamma$ and $\Gamma^{*}$. This work is currently being carried out by Professor Warschawski and myself. In the case of symmetric regions, the use of circular arcs orthogonal to the graphs of $\varphi_{-}$and $\varphi_{+}$ leads to a relatively simple derivation of the estimates in the remarkable, though difficult, paper of Gol'dberg-Stročik [1]. New estimates of wider applicability have been obtained by using other curve families. We are planning a joint publication on these topics in the near future.

\section{REFERENCES}

Accola, R. D. M.

[1] Differentials and extremal length on Riemann surfaces, Proc. Nat. Acad. Sci. U.S.A. 46 (1960), 540-543. MR 22 \#9598.

Adams, D. R.

[1] (with Meyers, N. G.) Thinness and Weiner criteria for non-linear potentials, Indiana Univ. Math. J. 22 (1972), 169-197.

[2] The equivalence of two definitions of capacity, Proc. Amer. Math. Soc. 37 (1973), 529-533.

Ahlfors, L. V.

[1] Untersuchungen zur Theorie der konformen abbildungen und der ganzen Funktionen, Acta Soc. Sci. Fenn. Ser. A I, No. 9 (1930), 40 pp.

[2] (with Beurling, A.) Invariants conformes et problèmes extrémaux, C. R. Dixième Congrès Math. Scandinaves 1946, Jul. Gjellerups Forlag, Copenhagen, 1947, pp. 341-351. MR 9, 23.

[3] (with Beurling, A.) Conformal invariants. Construction and applications of conformal maps, Appl. Math. Ser., no. 18, Nat. Bureau of Standards, U.S. Government Printing Office, Washington, D.C., 1952. MR 14, 861.

[4] (with Beurling, A.) Conformal invariants and function-theoretic null-sets, Acta Math. 83 (1950), 101-129. MR 12, 171.

[5] (with Sario, L.) Riemann surfaces, Princeton Math. Ser., no. 26, Princeton Univ. Press, Princeton, N.J., 1960. MR 22 \#5729.

[6] Classical and contemporary analysis, SIAM Rev. 3 (1961), 1-9. MR 23 \#A291.

[7] Geodesic curvature and area, Studies in Math. Anal. and Related Topics, Stanford Univ. Press, Stanford, Calif., 1962, pp. 1-7. MR 26 \#2598.

[8] Lectures on quasiconformal mappings, Van Nostrand Math. Studies, no. 10, Van Nostrand, Princeton, N.J., 1966. MR 34 \#336. 
[9] Conformal invariants: Topics in geometric function theory, McGraw-Hill, New York, 1973.

Akaza, T.

[1] (with Oikawa, K.) Examples of weak boundary components, Nagoya Math. J. 18 (1961), 165-170. MR 24 \#A1387.

Besicovitch, A. S.

[1] On two problems of Loewner, J. London Math. Soc. 27 (1952), 141-144. MR 13, 831 .

Beurling, A.

[1-3] Cf. Ahlfors, L. V.; Beurling, A. [2-4]

Blatter, C.

[1] Über Extremallängen auf geschlossen Flächen, Comment. Math. Helv. 35 (1961), 153-168. MR 24 \#A1389.

[2] Zur Riemannschen Geometrie in Grossen auf dem Möbiusband, Compositio Math. Brelot, $\mathrm{M}$. 15 (1961), 88-107. MR 25 \#3484.

[1] On topologies and boundaries in potential theory, Lecture Notes in Math., vol. Chern, S. S. 175, Springer-Verlag, Berlin and New York, 1971. MR 43 \#7654.

[1] (with Levine, H. I.; Nirenberg, L.) Intrinsic norms on a complex manifold, Global Analysis (Papers in Honor of K. Kodaira), Univ. of Tokyo Press, Tokyo, 1969, pp. 119-139. MR 40 \#8084.

Choquet, G.

[1] Forme abstraite du théorème de capacitabilité, Ann. Inst. Fourier (Grenoble) 9 (1959), 83-89. MR 22 \#3692b.

Constantinescu, C. and Cornea, A.

[1] Ideale Ränder Riemannscher Flächen, Ergebnisse der Mathematik und ihrer Doob, J. L. Grenzgebiete, N. F., Band 32, Springer-Verlag, Berlin, 1963. MR 28 \#3151.

[1] Boundary properties of functions with finite Dirichlet integrals, Ann. Inst. Fourier (Grenoble) 12 (1962), 573-621. MR 30 \#3992.

Fuchs, W. H. J.

[1] Topics in the theory of functions of one complex variable, Van Nostrand Math. Studies, no. 12, Van Nostrand, Princeton, N.J., 1967. MR 36 \#3954.

Fuglede, B.

[1] Extremal length and functional completion, Acta Math. 98 (1957), 171-219. MR Gaier, D. 20 \#4187.

[1] Estimates of conformal mappings near the boundary, Indiana Univ. Math. J. 21 (1971/72), 581-595. MR 45 \#2151.

Gal, I. S.

[1] Conformally invariant metrics on Riemann surfaces, Proc. Nat. Acad. Sci. U.S.A. 45 (1959), 1629-1633. MR 22 \#3810.

[2] Conformally invariant metrics and uniform structures. I, II, Nederl. Akad. Wetensch. Proc. Ser. A 63=Indag. Math. 22 (1960), 218-231, 232-244. MR 22 \#9597.

Gehring, F. W.

[1] (with Väisälä, J.) On the geometric definition for quasi-conformal mappings, Comment. Math. Helv. 36 (1961), 19-32. MR 25 \#4099.

[2] Symmetrization of rings in space, Trans. Amer. Math. Soc. 101 (1961), 499-519. MR 24 \#A2677. 
[3] Extremal length definitions for the conformal capacity of rings in space, Michigan Math. J. 9 (1962), 137-150. MR 25 \#4098.

[4] Quasiconformal mappings in space, Bull. Amer. Math. Soc. 69 (1963), 146-164. MR 26 \#2606.

[5] (with Väisälä, J.) The coefficients of quasiconformality of domains in space, Acta Math. 114 (1965), 1-70. MR 31 \#4905.

[6] Definitions for a class of plane quasiconformal mappings, Nagoya Math. J. 29 (1967), 175-184. MR 35 \#4410.

[7] Inequalities for condensers, hyperbolic capacity, and extremal lengths, Michigan Math. J. 18 (1971), 1-20. MR 44 \#2915.

Grötzsch, $\mathrm{H}$.

[1-11] Eleven papers in Ber. Verh. Sächs. Akad. Wiss. Leipzig Math. Phys. (19281932).

[12] Über zwei Verschiebungsprobleme der konformen Abbildung, Sitzgsber. Preuss. Akad. Wiss. Phys.-Math. 1933, 87-100.

[13-15] Über die Geometrie der schlichten konformen Abbildung. I, II, III, Sitzgsber. Preuss. Akad. Wiss. Phys.-Math. 1933, 654-671, 893-908; ibid. 1934, 434-444. Gol'dberg, A. A.

[1] (with Stročik, T. V.) Conformal mapping of symmetric half-strips and angular regions, Litovsk. Mat. Sb. 6 (1966), 227-239. (Russian) MR 35 \#5593.

Havin, V. P.

[1] Cf. Maz'ja, V. G.; Havin, V. P. [1]

Hersch, J.

[1] (with Pfluger, A.) Principe de l'augmentation des longueurs extrémales, C. R. Acad. Sci. Paris 237 (1953), 1205-1207. MR 15, 301.

[2] Longueurs extrémales et géométrie globale, Ann. Sci. École Norm. Sup. (3) 72 (1955), 401-414. MR 18, 146.

[3] Longueurs extrémales et théorie des fonctions, Comment. Math. Helv. 29 (1955), 301-337. MR 17, 835.

Jenkins, J. A.

[1] Some problems in conformal mapping, Trans. Amer. Math. Soc. 67 (1949), 327-350. MR 11, 341.

[2] Some results related to extremal length, Contributions to the Theory of Riemann Surfaces, Ann. of Math. Studies, no. 30, Princeton Univ. Press, Princeton, N.J., 1953, pp. 87-94. MR 15, 115.

[3] On the existence of certain general extremal metrics, Ann. of Math. (2) 66 (1957), 440-453. MR 19, 845.

[4] Univalent functions and conformal mapping, Ergebnisse der Măthematik und ihrer Grenzgebiete, N.F., Heft 18, Reihe: Moderne Funktionentheorie, SpringerVerlag, Berlin, 1958. MR 20 \#3288.

[5] (with Oikawa, K.) On the growth of slowly increasing unbounded harmonic functions, Acta Math. 124 (1970), 37-63. MR 41 \#3789.

[6] (with Oikawa, K.) On results of Ahlfors and Hayman, Illinois J. Math. 15 (1971), 664-671. MR 45 \#5332.

Jurchescu, $M$.

[1] Modulus of a boundary component, Pacific J. Math. 8 (1958), 791-804. MR 21 \#2047.

Klein, Felix

[1] On Riemann's theory of algebraic functions and their integrals. A supplement to the usual treatises, Dover, New York, 1963. MR 28 \#1295. 
Keen, L.

[1] An extremal length on a torus, J. Analyse Math. 19 (1967), 203-206. MR 35 \#7269.

Kusunoki, Y.

[1] (with Mori, S.) Some remarks on boundary values of harmonic functions with finite Dirichlet integrals, J. Math. Kyoto Univ. 7 (1967), 315-324. MR 37 \#1589.

[2] On some boundary properties of harmonic Dirichlet functions, Proc. Japan Acad. 46 (1970), 277-282. MR 45 \#3695.

Lehto, $\mathrm{O}$

[1] (with Virtanen, K. I.) Quasikonforme Abbildungen, Die Grundlehren der math. Wissenschaften in Einzeldarstellungen mit besonderer Berücksichtigung der Anwendungsgebiete, Band 126, Springer-Verlag, Berlin and New York, 1965. MR 32 \#5872.

Levine, H. I.

[1] Cf. Chern, S. S.; Levine, H. I.; Nirenberg, L. [1]

Lions, J. L.

[1] (with Magenes, E.) Non-homogeneous boundary value problems and applications, Springer-Verlag, Berlin and New York, 1972.

Magenes, E.

[1] Cf. Lions, J. L.; Magenes, E. [1]

Marden, A.

[1] The weakly reproducing differentials on open Riemann surfaces, Ann. Acad. Sci. Fenn. Ser. A I No. 359 (1965), 32 pp. MR 32 \#5868.

[2] (with Rodin, B.) A complete extremal distance problem on open Riemann surfaces, Bull. Amer. Math. Soc. 72 (1966), 326-328. MR 35 \#3056.

[3] (with Rodin, B.) Extremal and conjugate extremal distance on open Riemann surfaces with applications to circular-radial slit mappings, Acta Math. 115 (1966), 237-269. MR 34 \#2862.

[4] (with Rodin, B.) Periods of differentials on open Riemann surfaces, Duke Math. J. 33 (1966), 103-108. MR 33 \#4260.

Maz'ja, V. G.

[1] (with Havin, V. P.) A nonlinear analogue of the Newtonian potential and metric properties of the $(p, 1)$-capacity, Dokl. Akad. Nauk SSSR 194 (1970), 770-773= Soviet Math. Dokl. 11 (1970), 1294-1298. MR 42 \#7926.

Meyers, N. G.

[1] Cf. Adams, D. R.; Meyers, N. G. [1]

Minda, C. D.

[1] Extremal length and harmonic functions on Riemann surfaces, Trans. Amer. Math. Soc. 171 (1972), 1-22.

[2] Extremal length and reproducing differentials on Riemann surfaces (to appear).

Mizumoto, $\mathrm{H}$.

[1] Theory of Abelian differentials and relative extremal length with applications to extremal slit mappings, Japan. J. Math. 37 (1968), 1-58. MR 39 \#7090.

[2] Periods of differentials and relative extremal length. I, II, Kōdai Math. Sem. Rep. 21 (1969), 205-222, 399-404. MR 40 \#340; 41 \#7095.

Mori, S.

[1] Cf. Kusunoki, Y.; Mori, S. [1]

Nakai, $M$.

[1] Cf. Sario, L.; Nakai, M. [7]

Nevanlinna, R.

[1] Polygonal representation of Riemann surfaces, Lectures on Functions of a 
Complex Variable, Univ. of Michigan Press, Ann. Arbor, Mich., 1955, pp. 65Nirenberg, $\mathrm{L}$. 70. MR 16, 1097.

[1] Cf. Chern, S. S.; Levine, H. I.; Nirenberg, L. [1]

Obrock, A. E.

[1] On bounded oscillation and asymptotic expansion of conformal strip mappings, Trans. Amer. Math. Soc. 173 (1972), 183-201.

Ohtsuka, M.

[1] Dirichlet problems on Riemann surfaces and conformal mappings, Nagoya Math. J. 3 (1951), 91-137. MR 13, 642.

[2] Sur un théorème étoile de Grosś, Nagoya Math. J. 9 (1955), 191-207. MR 17, 1191.

[3] Extremal length of families of parallel segments, J. Sci. Hiroshima Univ. Ser. A I Math. 28 (1964), 39-51. MR 29 \#3666.

[4] Extremal length of level surfaces and orthogonal trajectories, J. Sci. Hiroshima Univ. Ser. A I Math. 28 (1964), 259-270. MR 31 \#1389.

[5] On limits of BLD functions along curves, J. Sci. Hiroshima Univ. Ser. A I Math. 28 (1964), 67-70. MR 29 \#6012.

[6] On weak and unstable components, J. Sci. Hiroshima Univ. Ser. A I Math. 28 (1964), 53-58. MR 29 \#3667.

[7] A computation of extremal length in an abstract space, J. Sci. Hiroshima Univ. Ser. A I Math. 29 (1965), 87-96. MR 32 \#5841.

[8] Dirichlet principle on Riemann surfaces, J. Analyse Math. 19 (1967), 295-311. MR 36 \#1638.

[9] Dirichlet problem, extremal length, and prime ends, Van Nostrand Reinhold, New York, 1970.

[10] Extremal length and precise functions in 3-space (to appear).

Oikawa, K.

[1] On a criterion for the weakness of an ideal boundary component, Pacific J. Math. 9 (1959), 1233-1238. MR 22 \#100.

[2] On the stability of boundary components, Pacific J. Math. 10 (1960), 263-294. MR 27 \#1590.

[3] Cf. Akasa, T.; Oikawa, K. [1]

[4] Welding of polygons and the type of Riemann surfaces, Kodai Math. Sem. Rep. 13 (1961), 37-52. MR 23 \#A3253.

[5] $A$ remark to the construction of Riemann surfaces by welding, J. Sci. Hiroshima Univ. Ser. A I Math. 27 (1963), 213-216. MR 29 \#241.

[6] (with Suita, N.) On parallel slit mappings, Kodai Math. Sem. Rep. 16 (1964), 249-254. MR 30 \#1237.

[7] Remarks to Conformal mappings onto radially slit disks, Sci. Papers College Gen. Ed. Univ. Tokyo 15 (1965), 99-109. MR 33 \#1438.

[8] Cf. Tamura, J.; Oikawa, K.; Yamazaki, K. [1]

[9] Cf. Sario, L.; Oikawa, K. [6]

[10] (with Suita, N.) On conformal mappings into incised radial slit disks, Kōdai Math. Sem. Rep. 22 (1970), 45-52. MR 44 \#1798.

[11] Cf. Jenkins, J. A.; Oikawa, K. [5]

[12] Cf. Jenkins, J. A.; Oikawa, K. [6]

Ozawa, M.

[1] Classification of Riemann surfaces, Kōdai Math. Sem. Rep. 1952, 63-76. MR 14, 462. 
Pfluger, A.

[1] Cf. Hersch, J.; Pfluger, A. [1]

[2] Extremallängen und Kapazität, Comment. Math. Helv. 29 (1955), 120-131. MR 16, 810.

[3] Theorie der Riemannschen Flächen, Springer-Verlag, Berlin, 1957. MR 18, 796. $\mathrm{Pu}, \mathrm{P} . \mathrm{M}$.

[1] Some inequalities in certain nonorientable Riemannian manifolds, Pacific J. Math. 2 (1952), 55-71. MR 14, 87.

Rengel, E.

[1] Über einige Schlitz-Theoreme der konformen abbildung, Schr. Math. Sem. Inst. Angew. Math. Univ. Berlin 1 (1933), 141-162.

Renggli, $\mathbf{H}$.

[1] Quasiconformal mappings and extremal lengths, Amer. J. Math. 86 (1964), 63-69. MR 30 \#1244.

Rodin, B.

[1] Extremal length of weak homology classes on Riemann surfaces, Proc. Amer. Math. Soc. 15 (1964), 369-372. MR 29 \#240.

[2-3] Cf. Marden, A.; Rodin, B. [2-3]

[4] Extremal length and removable boundaries of Riemann surfaces, Bull. Amer. Math. Soc. 72 (1966), 274-276. MR 32 \#4265.

[5] Cf. Marden, A.; Rodin, B. [4]

[6] (with Sario, L.) Principal functions, Van Nostrand, Princeton, N.J., 1968. MR 37 \#5378.

[7] Extremal length and geometric inequalities, Proc. Sympos. Pure Math., vol. 11, Amer. Math. Soc., Providence, R.I., 1968, pp. 370-376. MR 38 \#6052.

Royden, H. L.

[1] Open Riemann surfaces, Ann. Acad. Sci. Fenn. Ser. A I No. 249/5 (1958), 13 pp. Sario, L. MR 20 \#7102.

[1] Über Riemannsche Flächen mit hebbarem Rand, Ann. Acad. Sci. Fenn. Ser. A I Math.-Phys. No. 50 (1948), 79 pp. MR 10, 365.

[2] Capacity of the boundary and of a boundary component, Ann. of Math. (2) 59 (1954), 135-144. MR 15, 518.

[3] Strong and weak boundary components, J. Analyse Math. 5 (1956/57), 389-398. MR 20 \#979.

[4] Cf. Ahlfors, L. V.; Sario, L. [5]

[5] Cf. Rodin, B.; Sario, L. [6].

[6] (with Oikawa, K.) Capacity functions, Die Grundlehren der math. Wissenschaften, Band 149, Springer-Verlag, New York, 1969. MR. 40 \#7441.

[7] (with Nakai, M.) Classification theory of Riemann surfaces, Die Grundlehren der math. Wissenschaften, Band 164, Springer-Verlag, Berlin and New York, 1970. MR 41 \#8660.

Schlesinger, E.

[1] Conformal invariants and prime ends, Amer. J. Math. 80 (1958), 83-102. MR 20 \#1775.

Strebel, K.

[1] Eine Ungleichung für Extremale Längen, Ann. Acad. Sci. Fenn. Ser. A I Math.Phys. No. 90 (1951), 8 pp. MR 13, 338.

[2] A remark on the extremal distance of two boundary components, Proc. Nat. Acad. Sci. U.S.A. 40 (1954), 842-844. MR 16, 917. 
[3] Die extremale Distanz zweier Enden einer Riemannschen Fläche, Ann. Acad. Sci. Fenn. Ser. A I No. 179 (1955), 21 pp. MR 16, 917.

Stročik, T. V.

[1] Cf. Gol'dberg, A. A.; Stročik, T. V. [1]

[2] On conformal mapping of half-strips of constant width, Ukrain. Mat. $\check{Z} .21$ (1969), 60-72=Ukrainian Math. J. 21 (1969),48-57. MR 39 \# 7076.

[3] On conformal mapping of a class of half-strips, Sibirsk. Mat. Z. 11 (1970), 859_ 878=Siberian Math. J. 11 (1970), 647-661. MR 42 \#7874. Suita, N.

[1] Cf. Oikawa, K.; Suita, N. [6]

[2] Minimal slit domains and minimal sets, Kōdai Math. Sem. Rep. 17 (1965), 166186. MR 32 \#4258.

[3] On radial slit disc mappings, Kōdai Math. Sem. Rep. 18 (1966), 219-228. MR 35 \#356.

[4] On slit rectangle mappings and continuity of extremal length, Ködai Math. Sem. Rep. 19 (1967), 425-438. MR 38 \#1241.

[5] On circular and radial slit disc mappings, Kōdai Math. Sem. Rep. 20 (1968), 127-145. MR 38 \#3416.

[6] Capacitability and extremal radius, Kōdai Math. Sem. Rep. 20 (1968), 442-447. MR 39 \#2962.

[7] On continuity of extremal distance and its applications to conformal mappings, Kōdai Math. Sem. Rep. 21 (1969), 236-251. MR 41 \#3734.

[8] Cf. Oikawa, K.; Suita, N. [10]

Tamura, J.

[1] (with Oikawa, K.; Yamazaki, K.) Examples of minimal parallel slit domains, Proc. Amer. Math. Soc. 17 (1966), 283-284. MR 32 \#4260.

Teichmüller, $\mathbf{O}$.

[1] Untersuchungen über konforme und quasikonforme Abbildungen, Deutsche Math. 3 (1938), 621-678.

[2] Eine Verschärfung des Dreikreisesatzes, Deutsche Math. 4 (1939), 16-22.

[3] Extremale quasikonforme Abbildungen und quadratische Differentiale, Abh. Preuss. Akad. Wiss. Math.-Nat. 22 (1939), 1-197.

[4] Über Extremalprobleme der konformen Geometrie, Deutsche Math. 6 (1941), 50-77.

Tsuji, M.

[1] Potential theory in modern function theory, Maruzen, Tokyo, 1959. MR 22 \#5712. Väisälä, J.

[1] Cf. Gehring, F. W.; Väisälä, J. [1]

[2] On quasiconformal mappings in space, Ann. Acad. Sci. Fenn. Ser. A I No. 298 (1961), 36 pp. MR 25 \#4100a.

[3] On the null-sets for extremal distances, Ann. Acad. Sci. Fenn. Ser. A I No. 332 (1962), 12 pp. MR 26 \#5148.

[4] Cf. Gehring, F. W.; Väisälä, J. [5]

Virtanen, K. I.

[1] Cf. Lehto, O.; Virtanen, K. K. [1]

Warschawski, S. E.

[1] Zur Randverzerrung bei konformer Abbildung, Compositio Math. 1 (1935), 314343.

[2] On the preservation of angles at a boundary point in conformal mapping, Bull. Amer. Math. Soc. 42 (1936), 674-680. 
[3] On conformal mapping of infinite strips, Trans. Amer. Math. Soc. 51 (1942) 280-335. MR 4, 9.

Weill, G. G.

[1] Reproducing kernels and orthogonal kernels for analytic differentials on Riemann surfaces, Pacific J. Math. 12 (1962), 729-767. MR 27 \#292.

Wolontis, V.

[1] Properties of conformal invariants, Amer. J. Math. 74 (1952), 587-606. MR 14, 36.

Yamaguchi, $\mathrm{H}$.

[1] Regular operators and spaces of harmonic functions with finite Dirichlet integra, on open Riemann surfaces, J. Math. Kyoto Univ. 8 (1968), 169-198. MR 38 \#1254.

Yamazaki, K.

[1] Cf. Tamura, J.; Oikawa, K.; Yamazaki, K. [1]

Yoshida, $M$.

[1] Generalized bilinear relations on open Riemann surfaces, J. Math. Kyoto Univ. 8 (1968), no. 2.

Ziemer, W.

[1] Extremal length and conformal capacity, Trans. Amer. Math. Soc. 126 (1967), 460-473. MR 35 \#1776.

Current address: Department of Mathematics, University of California at San Diego, La Jolla, California 92037 